\title{
Involvement of RQCD1 overexpression, a novel cancer-testis antigen, in the Akt pathway in breast cancer cells
}

\author{
MASAHIKO AJIRO $^{1}$, TOYOMASA KATAGIRI ${ }^{1,2}$, KOJI UEDA $^{3}$, HIDEWAKI NAKAGAWA $^{3}$, \\ CHIKAKO FUKUKAWA ${ }^{1}$, MENG-LAY LIN ${ }^{1}$, JAE-HYUN PARK ${ }^{1}$, TOSHIHIKO NISHIDATE ${ }^{1}$, \\ YATARO DAIGO $^{1}$ and YUSUKE NAKAMURA ${ }^{1}$
}

\begin{abstract}
${ }^{1}$ Laboratory of Molecular Medicine, Human Genome Center, Institute of Medical Science, The University of Tokyo, Tokyo; ${ }^{2}$ Division of Genome Medicine, Institute for Genome Research, The University of Tokushima, Tokushima;

${ }^{3}$ Laboratory for Biomarker Development, Center for Genomic Medicine, RIKEN, Yokohama, Japan
\end{abstract}

Received May 25, 2009; Accepted July 2, 2009

DOI: 10.3892/ijo_00000379

\begin{abstract}
We here report identification and characterization of Required for Cell Differentiation 1 homolog (RQCD1) as a potential therapeutic target for breast cancer. Gene-expression profiling analysis of breast cancer cells, semi-quantitative RT-PCR, Northern blotting and Western blotting confirmed RQCD1 to be frequently up-regulated in breast cancer specimens and breast cancer cell lines. On the other hand, its expression was very weak or hardly detectable in normal human tissues except testis, indicating this molecule to be a novel cancer-testis antigen. Treatment of breast cancer cell lines with siRNA targeting RQCD1 drastically suppressed cell proliferation. Concordantly, introduction of exogenous RQCD1 into HEK293 cells significantly enhanced cell growth, implying RQCD1 to have an oncogenic activity. Coimmunoprecipitation experiments and immunocytochemical staining revealed an interaction of RQCD1 protein with Grb10 interacting GYF protein 1 (GIGYF1) and 2 (GIGYF2) proteins, involved in regulation of Akt activation, in breast cancer cells. Interestingly, knockdown of either of $R Q C D 1$, GIGYF1 or GIGYF2 resulted in significant reduction of the phosphorylation of Akt at Ser 473 in breast cancer cell lines. Our findings suggest that RQCD1 is a potential molecular target for treatment of breast cancer.
\end{abstract}

\section{Introduction}

Breast cancer is the most frequent cancer among women worldwide, ranking as first in incidence and fifth in mortality by global statistics in 2002. Although the incidence of breast

Correspondence to: Dr Yusuke Nakamura, Laboratory of Molecular Medicine, Human Genome Center, Institute of Medical Science, The University of Tokyo, 4-6-1 Shirokanedai, Minato-ku, Tokyo 108-8639, Japan

E-mail: yusuke@ims.u-tokyo.ac.jp

Key words: breast cancer, molecular target, Akt-signal cancer has been significantly increasing, its mortality rate has been reduced and quality of life (QOL) of the patients has been significantly improved in most developed countries probably due to widespread mammographic screening as well as development of molecular targeting drugs like tamoxifen, aromatase inhibitor and trastuzumab $(1,2)$. However, benefits of such molecular targeting drugs are still very limited to the patients at an advanced stage or those with a triple-negative breast cancer. In addition, we often observe adverse reactions caused by these drugs; for instance, increase in the risk of endometrial cancer in patients having long-term tamoxifen administration and that of severe cardiac toxicity with trastuzumab treatment as well as the risk of bone fracture due to osteoporosis in postmenopausal women with aromatase inhibitor prescription are recognized as adverse effects (3). Hence, to overcome such adverse effects and to further improve the patients' QOL, development of novel molecular targeting drugs to provide better clinical management is essentially important.

Genome-wide gene expression profiling analyses by cDNA microarray technology was shown to be an effective approach for discovery of target molecules for various types of cancer $(4,5)$. In an attempt to identify novel molecular targets in breast cancer, we previously performed genomewide expression profiling analysis of breast cancer specimens by cDNA microarray (6), and identified a number of genes that play important roles in the growth of breast cancer cells (7-13). Such molecules are considered to be potential molecular targets for development of new therapeutic modalities against breast cancer.

We report the identification and characterization of $R Q C D 1$, which was significantly up-regulated in breast cancer cells. A homolog of this gene was identified as a regulator for cell differentiation in nitrogen-starved fission yeast (14) and mammalian RQCD1 was later indicated to function as a transcriptional cofactor that was involved in retinoic acid-induced differentiation of mouse teratocarcinoma cell line F9 (15). However, its role in human carcinogenesis has not been described so far.

Here we report involvement of RQCDI overexpression in breast cancer cells and its interaction with GIGYF1 and 
GIGYF2 proteins, which were previously reported to have critical roles in phosphatidylinositol-3-kinase (PI3K)/Akt signaling pathway (16). Our findings suggest that RQCD1 might be a potential molecular target for the development of anticancer drugs to breast cancer.

\section{Materials and methods}

Breast cancer cell lines and clinical samples. Human breast cancer cell lines, HCC-1937, BT-549, MCF-7, BSY-1, MDAMB-435S, SKBR-3, T-47D, MDA-MB-231 and YMB-1, human normal ductal epithelial cell MCF10A, human embryonic kidney cell lines, HEK293 and HEK293T, were purchased from American Type Culture Collection (ATCC; Rockville, MD, USA). They were cultured under the recommendations of their respective depositors. HBC-4 and HBC-5 cell lines were kindly provided by $\mathrm{Dr}$ Takao Yamori of Division of Molecular Pharmacology, Cancer Chemotherapy Center, Japanese Foundation for Cancer Research. Culture media for these cell lines were as follows, RPMI-1640 (Invitrogen, Carlsbad, CA, USA) for HCC-1937, T-47D, SKBR-3, YMB-1, HBC-4 and HBC-5; EMEM (Invitrogen) with $0.001 \%$ insulin for MCF-7 and HEK293; L-15 (SigmaAldrich, St Louis, MO, USA) for MDA-MB-231 and MDAMB-435S; DMEM (Sigma-Aldrich) for HEK293T; MEGM (Lonza, Basel, Switzerland) with $13 \mathrm{mg} / \mathrm{ml}$ Bovine Pituitary Extract, $0.5 \mathrm{mg} / \mathrm{ml}$ hydrocortisone, $10 \mu \mathrm{g} / \mathrm{ml} \mathrm{EGF,} 5 \mathrm{mg} / \mathrm{ml}$ insulin and $100 \mathrm{ng} / \mathrm{ml}$ cholera toxin (Lonza) for MCF10A. Each medium except MEGM was supplemented with $10 \%$ fetal bovine serum (Cansera International, Ontario, Canada) and $1 \%$ antibiotic/antimycotic solution (Sigma-Aldrich). MDA-MB-231 and MDA-MB-435S cells were maintained at $37^{\circ} \mathrm{C}$ in atmosphere of humidified air without $\mathrm{CO}_{2}$. Other cell lines were maintained at $37^{\circ} \mathrm{C}$ in atmosphere of humidified air with $5 \% \mathrm{CO}_{2}$. Tissue samples from surgically resected breast cancers and their corresponding clinical information were obtained from Department of Breast Surgery, Cancer Institute Hospital, Tokyo after obtaining written informed consent. This study including the use of all clinical materials described above was approved by individual institutional Ethics Committees.

Semiquantitative reverse transcription-PCR analysis. We extracted total RNAs from 12 microdissected clinical breast cancer cells and culture cell lines using RNeasy kit according to the manufacturer's protocol (GE Healthcare, Buckinghamshire, UK). Extracted RNAs and normal human tissue polyadenylate RNAs were treated with DNase I (Nippon Gene, Tokyo, Japan), and then were reversely transcribed using SuperScript First-Strand Synthesis System (Invitrogen). We prepared appropriate dilutions of each single-stranded cDNA for subsequent PCR by monitoring $\beta$-actin (ACTB) as an internal control. The PCR primer sequences were as follows; 5'-GGAACGGTGAAGGTGACAGC-3' and 5'-ACC TCCCCTGTGTGGACTTG-3' for ACTB, 5'-GGACTTGTT AGTTGGCTTCTGTC-3' and 5'-GATCACTTCTCTTCA GGCTTGC-3' for 3'-UTR region of RQCD1 (analysis of expression in clinical samples and cell lines). 5'-CTGGCA CAAGTGGATAGAGAAA-3' and 5'-CAGAAGGCTCTT TGGATAGCTG-3' for RQCD1 (analysis of its knockingdown effect), 5'-CAGCAGAGACACTCAACTTTGG-3' and
5'-CTTCTTCGATGCTTCTTTGGTAA-3' for GIGYF1, and 5'-CGGCAGAGAAGAAATGTTAGC-3' and 5'-GCTTTC TCCCTACTGATGTTGG-3' for GIGYF2.

5' and 3' rapid amplification of cDNA ends (5' and 3' RACE). The 5' and 3' RACE experiments were carried out using SMART RACE cDNA Amplification kit (Takara Clontech) according to the manufacturer's instructions. For the amplification of the 5' and 3 ' sequences of RQCD1 cDNA, genespecific primers (5'-GCGGCAACCCTGTAATTCCCATA GAC-3' for 5' RACE and 5'-GGAGGTGCTTGGGATTAA GGTGACAG-3' for 3' RACE) and a universal primer mixture supplied in the kit were used. The cDNA template was synthesized from mRNA purified from HBC-4 breast cancer cells, using Superscript II Reverse Transcriptase (Invitrogen). The PCR products were cloned using TA cloning kit (Invitrogen) and sequences were determined by DNA sequencing (ABI3700; PE Applied Biosystems, Foster, CA).

Northern blotting. Breast cancer Northern blot membranes were prepared as described previously (7). Human MultipleTissue Northern blot membrane (Takara Clontech) and breast cancer Northern blot membranes were hybridized with $\left[\alpha^{32} \mathrm{P}\right]$-dCTP labeled cDNA probe for $R Q C D 1$, prepared by RT-PCR (see below) with megaprime DNA labeling system (GE Healthcare). Prehybridization, hybridization and washing were performed as described previously (17). The blots were autoradiographed with intensifying screens at $-80^{\circ} \mathrm{C}$ for 14 days. Specific probe for $R Q C D 1$ (283 bp) was prepared by RT-PCR using the primer set of 5'-GGACTTGTTAGT TGGCTTCTGTC-3' and 5'-GATCACTTCTCTTCAGGCT TGC-3'.

Construction of expression vectors. To expression vector constructs for RQCD1, GIGYF1 and GIGYF2, each entire coding sequence was amplified by PCR using KOD-Plus DNA polymerase (Toyobo, Osaka, Japan). Primer sets were as follows; 5'-GGAATTCAATGCACAGCCTGGCGACGG-3' and 5'-GGACTCGAGCTGAGGGGGCAGGGGGATA-3' for $R Q C D 1,5$ '-GTTAAGTAGCGGCCGCTCATGGCA GCAGAGACACTCAAC-3' and 5'-CCGCTCGAGGTAGTC ATCCACGCTCTC-3' for GIGYF1, and 5'-GTTAAGTAG CGGCCGCTCATGGCAGCGGAAACGCAGAC-3' and 5'-C CGCTCGAGGTAGTCATCCAACGTCTC-3' for GIGYF2 (underlines indicate recognition sites of restriction enzymes). The PCR products were inserted into pCAGGSn3FC or pCAGGSnHC expression vector in frame with hemagglutinin (HA)-tag or Flag-tag at the C-terminus, respectively. DNA sequences of each construct were confirmed by DNA sequencing (ABI3700; PE Applied Biosystems).

Preparation of anti-RQCD1 polyclonal antibody. Plasmid designed to express the full-length RQCD1 with glutathione $S$-transferase (GST)-tag at the N-terminus was constructed using pGEX-6P-1 vector (GE Healthcare). The recombinant RQCD1 protein was expressed in BL21-CodonPlus-RIL Escherichia coli strain (Stratagene, La Jolla, CA, USA) and purified using Glutathione Sepharose 4B (Zymed Laboratories, South San Francisco, CA, USA), followed by digestion with PreScission Protease (GE Healthcare) to remove GST-tag 
according to the supplier's protocols. The purified recombinant protein was used for immunization of rabbits (SCRUM, Tokyo, Japan). The immune sera were subsequently purified on antigen affinity columns using Affi-gel 10 (Bio-Rad Laboratories, Hercules, CA, USA) according to supplier's instructions.

Western blotting. To examine the expression of RQCD1 protein in breast cancer and normal tissues, we used Protein Medley (Takara Clontech) tissue lysates for human normal mammary gland, lung, heart, liver and kidney. Cultured breast cancer cells were harvested with lysis buffer containing $25 \mathrm{mM}$ Tris-HCl (pH 7.4), 150 mM NaCl, 1 mM EDTA, 1\% NP-40, $10 \%$ glycerol, $1 \%$ Phosphatase Inhibitor Cocktail Set II and $0.1 \%$ Protease Inhibitor Cocktail Set III (Calbiochem, San Diego, CA). SDS-PAGE and Western blotting were performed as described previously (7). Antibodies used in this study were as follows; anti-RQCD1 rabbit polyclonal antibody $(0.6 \mu \mathrm{g} / \mathrm{ml})$ for RQCD1, anti-ß-actin mouse monoclonal antibody (Ac-15) (Sigma-Aldrich) $(10 \mathrm{ng} / \mathrm{ml})$ for ACTB, anti-Akt rabbit polyclonal antibody (\#9272) (Cell Signaling Technology, Danvers, MA, USA) (1:1,000 dilution) for Akt, anti-phospho Akt (Ser 473) mouse monoclonal antibody (\#4051) (Cell Signaling Technology) (1:1,000 dilution) for phosphorylated Akt on Ser 473, anti-HA rat monoclonal antibody (3F10) (Roche, Basel, Switzerland) (20 ng/ml) for HA-tag, anti-Flag mouse monoclonal antibody (M2) (SigmaAldrich) (25 ng/ml) for Flag-tag, and HRP-conjugated antimouse, rat or rabbit antibody (Santa Cruz Biotechnology, Santa Cruz, CA, USA) (40 ng/ml).

Immunocytochemical staining analysis. For immunocytochemical staining analysis, we seeded BT-549 breast cancer cells, at a density of $1 \times 10^{5}$ cells per chamber of a 2-well LabTek chamber slide (Nunc, Thermo Fisher Scientific, Waltham, MA, USA). After 24-h culture, cells were washed twice with phosphate-buffered saline (PBS) (-), fixed with $4 \%$ paraformaldehyde solution at $4^{\circ} \mathrm{C}$ for $15 \mathrm{~min}$, and then permealized with PBS (-) containing $0.1 \%$ Triton $\mathrm{X}-100$ at $4^{\circ} \mathrm{C}$ for $3 \mathrm{~min}$. Cells were covered with $5 \%$ bovine serum albumin (BSA) in PBS (-) at $4^{\circ} \mathrm{C}$ for 60 min to block non-specific binding before the primary antibody reaction. For the detection of endogenous RQCD1 in breast cancer cells, the cells were incubated with anti-RQCD1 rabbit polyclonal antibody $(6 \mu \mathrm{g} / \mathrm{ml})$ with $5 \%$ BSA for $60 \mathrm{~min}$ and subsequently Alexa 488 anti-rabbit IgG (4 $\mu \mathrm{g} / \mathrm{ml})$ (Molecular Probes, Eugene, OR, USA) with 5\% BSA for $60 \mathrm{~min}$. For the detection of exogenously expressed HA-tagged GIGYF1 and GIGYF2 proteins, cells were incubated with anti-HA rat monoclonal antibody (3F10) $(0.4 \mu \mathrm{g} / \mathrm{ml})$ (Roche) with 5\% BSA for $60 \mathrm{~min}$, and subsequently Alexa 594 anti-rat IgG diluted 1:500 in 5\% BSA for $60 \mathrm{~min}$. Then, the cells were mounted with VectaShield Mounting Medium with 4', 6-diamino-2'-phenylindole dihydrochloride (DAPI) (Vector Laboratories, Burlingame, CA, USA) to be counterstained their nuclei. Fluorescent images were obtained by TCS SP2 AOBS confocal microscope (Leica Microsystems, Wetzlar, Germany).

RNA interference assay. We generated the shRNA expression vectors against RQCD1 by cloning of double-stranded oligonucleotides into the BbsI site in the psiU6BX3.0 vector as describe previously (18). Cells $\left(1.0 \times 10^{6}\right)$ of BT-549 and HBC-4 cell lines were seeded in $10-\mathrm{cm}$ plates. Twenty-four hours after seeding, the cells were transfected with each of shRNA expression vectors targeting RQCDI (\#1 and \#2), or psiU6BX3.0 mock vector (without any insert) using FuGENE 6 transfection reagent (Roche) according to the manufacturer's instructions. Twenty-four hours after transfection, the cells were re-seeded to 6-Well Clear TC-Treated Microplate (Corning, Lowell, MA, USA) $\left(0.7 \times 10^{5}\right.$ cells/well) for cell proliferation and colony formation assays, and to $10-\mathrm{cm}$ plates $\left(3.5 \times 10^{5}\right.$ cells/plate) for RT-PCR and Western blotting with culture medium containing $0.5 \mathrm{mg} / \mathrm{ml}$ of geneticine (Invitrogen). After geneticine treatment for 7 days, the knockdown effect of shRNA was examined by semiquantitative RT-PCR and Western blotting analyses as described above. After geneticine treatment for 8 days, we performed cell proliferation assay with Cell Counting kit-8 (Dojindo, Kumamoto, Japan). Moreover, after geneticine treatment for 9 days, we performed colony formation assay by staining colonies with Giemsa staining solution (Merck, Whitehouse Station, NJ, USA) following fixation by $4 \%$ paraformaldehyde for $15 \mathrm{~min}$. For Akt activation analysis, we also used each of the oligo-duplex siRNAs (Sigma-Genosys, St Louis, MO, USA) targeting RQCD1 (\#1), GIGYF1 or GIGYF2, and also siRNA for EGFP as a control. Seventytwo hours after transfection of siRNAs, we evaluated Akt activity by Western blotting with anti-phospho Akt mouse monoclonal antibody which can recognize the phosphorylation of Akt at Ser 473 (\#4051; Cell Signaling Technology). To evaluate the effects on the level of Akt phosphorylation after knockdown of RQCD1, GIGYF1 or GIGYF2, the band intensities of Western blotting with anti-phospho-Akt and anti-Akt-antibodies were quantified by using Image $\mathbf{J}$ analysis software (http://rsb.info.nih.gov/ij) (19). The siRNA target sequences were as follows: 5'-GATCTATCAGTGGATC AAT-3' for RQCD1 (\#1), 5'-GATCTTGTTAGATGACACT-3' for RQCDI (\#2), 5'-CCTTCCGAAGGGCTAGAGG-3' for GIGYF1, 5'-CAAGATACCTTCAGACCTT-3' for GIGYF2, and 5'-GCAGCACGACTTCTTCAAG-3' for EGFP.

Preparation of RQCD1 stably-expressing cell lines. HEK293 cells were transfected with pCAGGS-HA-RQCD1 plasmid vector or mock plasmid vector using FuGENE 6 transfection reagent (Roche). Twenty-four hours after transfection, cells were incubated in culture medium with $0.5 \mathrm{mg} / \mathrm{ml}$ of Geneticine (Invitrogen) for 14 days. Then, more than 20 individual colonies were isolated, and then each colony was evaluated for its monoclonal expression of RQCD1 protein by immunocytochemical staining and Western blotting with anti-HA antibody. Finally, we established three independent clones and designated them as follows: HEK293-RQCD1-1, -2 and -3 (stable-1, -2 and -3 ), and HEK293-Mock-1, -2 and -3 (mock-1, -2 and -3). For cell proliferation assay, mock- and RQCD1-stable cell lines were seeded to collagen type 1 coated 6-well microplate (Asahi glass Co., Tokyo, Japan) $\left(0.4 \times 10^{5}\right.$ cells/well), and cell growth was evaluated with Cell Counting kit-8 (Dojindo) according to the manufacturer's instructions.

GST-pull down assay. HBC-4 was seeded at $1.0 \times 10^{6}$ cells in $10-\mathrm{cm}$ plate. Twenty-four hours after seeding, the cells were harvested with $500 \mu 1$ of ice-cold buffer containing $25 \mathrm{mM}$ 
Tris-HCl (pH 7.4), $150 \mathrm{mM} \mathrm{NaCl}, 1 \mathrm{mM}$ EDTA, 0.1\% NP-40, $10 \%$ glycerol, $1 \%$ Phosphatase Inhibitor Cocktail Set II and $0.1 \%$ Protease Inhibitor Cocktail Set III (Calbiochem), cleared by centrifugation at $18,000 \times \mathrm{g}$ for $15 \mathrm{~min}$ and rotated with $30 \mu \mathrm{l}$ of Sepharose-4B (Sigma-Aldrich) for $1 \mathrm{~h}$ at $4^{\circ} \mathrm{C}$ for pre-clear. Then, $1 \mu \mathrm{g}$ of GST alone or the same molecular number of the N-terminally GST-fused full-length RQCD1 recombinant protein was added to supernatants, and rotated for $1 \mathrm{~h}$ at $4^{\circ} \mathrm{C}$. Then, $10 \mu 1$ of Glutathione Sepharose-4B (Zymed Laboratories, South San Francisco, CA, USA) was added to each cell lysate and rotated for $1 \mathrm{~h}$ at $4^{\circ} \mathrm{C}$. Sepharose beads were washed with $500 \mu 1$ of lysis buffer for three times, and then bound proteins were eluted by addition of $30 \mu 1$ of SDS-PAGE sample buffer.

Mass spectrometric analysis. Eluted samples of GST-pull down assay were loaded onto SDS-PAGE followed by silver staining with SilverQuest Silver Staining kit (Invitrogen). The excised protein bands were reduced in $10 \mathrm{mM}$ Tris (2carboxyethyl) phosphine (Sigma-Aldrich) with $50 \mathrm{mM}$ ammonium bicarbonate (Sigma-Aldrich) for $30 \mathrm{~min}$ at $37^{\circ} \mathrm{C}$ and alkylated in $50 \mathrm{mM}$ iodoacetamide (Sigma-Aldrich) with $50 \mathrm{mM}$ ammonium bicarbonate for $45 \mathrm{~min}$ in the dark at $25^{\circ} \mathrm{C}$. Porcine trypsin (Promega, San Luis Obispo, CA) was added for a final enzyme: protein ratio of 1:20 and incubated at $37^{\circ} \mathrm{C}$ for $16 \mathrm{~h}$. The resulting peptide mixture was separated on a $100 \mu \mathrm{m}$ x $150 \mathrm{~mm}$ HiQ-Sil (KYA Technologies, Tokyo, Japan) using 30 min linear gradient from 5.4 to $29.2 \%$ acetonitrile in $0.1 \%$ trifluoroacetic acid (TFA) with total flow of $300 \mathrm{nl} / \mathrm{min}$. The eluting peptides were mixed with matrix solution $(4 \mathrm{mg} / \mathrm{ml} \alpha$-cyano-4-hydroxy-cinnamic acid, $0.08 \mathrm{mg} / \mathrm{ml}$ of ammonium citrate in $70 \%$ acetonitrile, $0.1 \%$ TFA) and automatically spotted onto MALDI target plates by MaP (KYA Technologies, Tokyo, Japan). Mass spectrometric analysis was performed on 4800 Plus MALDI/TOF/TOF Analyzer (Applied Biosystems/MDS Sciex). MS/MS peak list generated by the Protein Pilot version 2.0.1 software (Applied Biosystems/MDS Sciex) was exported to a local MASCOT version 2.2.03 search engine (Matrix Science, Boston, MA, USA) for protein data base search.

Co-immunoprecipitation assay. HEK293T cells were seeded at the density of $1.0 \times 10^{6}$ cells $/ 10-\mathrm{cm}$ plate. Twenty-four hours after seeding, the cells were transfected with indicated combinations of expression vectors. At $36 \mathrm{~h}$ after transfection, cells were harvested with $500 \mu 1$ of ice-cold buffer containing $25 \mathrm{mM}$ Tris- $\mathrm{HCl}$ (pH 7.4), $150 \mathrm{mM} \mathrm{NaCl}$, $1 \mathrm{mM}$ EDTA, 0.1\% NP-40, 10\% glycerol, 1\% Phosphatase Inhibitor Cocktail Set II and $0.1 \%$ Protease Inhibitor Cocktail Set III (Calbiochem), cleared by centrifugation at $18,000 \mathrm{x} \mathrm{g}$ at $4^{\circ} \mathrm{C}$ for $15 \mathrm{~min}$, and rotated with $10 \mu 1$ of anti-Flag (M2) agarose (Sigma-Aldrich) or anti-HA (HA-7) agarose (SigmaAldrich) at $4^{\circ} \mathrm{C}$ for $1 \mathrm{~h}$. Then, those agarose beads were washed with $500 \mu 1$ of washing buffer containing $25 \mathrm{mM}$ Tris- $\mathrm{HCl}$ (pH 7.4), $150 \mathrm{mM} \mathrm{NaCl}, 1 \mathrm{mM}$ EDTA, $0.1 \%$ NP-40, $10 \%$ glycerol for three times, and $500 \mu 1$ of buffer containing $25 \mathrm{mM}$ Tris- $\mathrm{HCl}$ (pH 7.4), $150 \mathrm{mM} \mathrm{NaCl}, 1 \mathrm{mM}$ EDTA. Finally, precipitated proteins were eluted by incubating with $20 \mu 1$ of $150 \mu \mathrm{g} / \mathrm{ml}$ 3XFlag peptide (Sigma-Aldrich) or $200 \mu \mathrm{g} / \mathrm{ml} \mathrm{HA}$ peptide (Sigma-Aldrich) at $4^{\circ} \mathrm{C}$ for $1 \mathrm{~h}$, respectively.
Statistical analysis. Statistical significance was examined by Student's t-test. A difference of $\mathrm{P}<0.05$ was considered to be statistically significant.

\section{Results}

RQCD1 is up-regulated in breast cancer cells. To screen molecules that could be applicable as targets for development of novel therapeutic drugs, we had carried out genome-wide expression profile analysis of 81 breast cancer specimens by cDNA microarray targeting 23,040 cDNAs or ESTs (6). Among dozens of up-regulated genes, we in this study focused on $R Q C D 1$, whose expression was frequently up-regulated (at least 3-fold more than normal ductal cells) in a solidtubular type of breast carcinoma. We confirmed its overexpression in 4 in 12 clinical solid-tubular cases by comparison with normal breast ductal cells or with whole mammary gland by semiquantitative RT-PCR (Fig. 1A). Subsequent Northern blot analysis using a RQCDI cDNA fragment revealed overexpression of its transcript (approximately $3.5 \mathrm{~kb}$ long) in breast cancer cell lines, while $R Q C D 1$ expression was very weak or hardly detectable in normal human organs except the testis (Fig. 1B) as concordant to the results of cDNA microarray analysis. Since the assembled cDNA sequence of $R Q C D 1$ (accession no. NM_005444; $900 \mathrm{bp}$ ) in the NCBI database was smaller than the size of the transcript indicated by Northern blot analysis, we performed the exonconnection, and 5' and 3' RACE experiments. We finally obtained the full-length cDNA sequences of human RQCDI consisting of 3,284 nucleotides (Genbank accession; AB500892) encoding a protein of 299 amino acids. The $R Q C D 1$ gene consists of eight exons and spans an approximately 45.4-kb genomic region on chromosomal band $2 \mathrm{q} 35$.

To investigate the expression of RQCD1 protein in breast cancer cells, we generated a polyclonal antibody against fulllength RQCD1 protein, and performed Western blotting analysis using the whole cell lysate from eight breast cancer cell lines as well as normal human tissues including mammary gland, lung, heart, liver and kidney. We detected a high level of RQCD1 protein in all the breast cancer cell lines examined, but its expression was hardly detectable in any of normal human tissues except the testis (Fig. 1C). Furthermore, we examined the subcellular localization of endogenous RQCD1 protein in the breast cancer cell line BT-549 by immunocytochemical staining analysis using the purified anti-RQCD1 polyclonal antibody. It was observed diffusely in both cytoplasm and nucleus of breast cancer cells (Fig. 1D).

Effect of RQCD1 on cell growth. To examine the functional role of RQCD1 in breast cancer cell growth, we knocked down the expression of endogenous RQCD1 in the breast cancer cell lines BT-549 and HBC-4, which showed high RQCD1 expression at both transcriptional and protein levels (Fig. 1), by means of small hairpin-RNA (shRNA) expression vector system. Semiquantitative RT-PCR and Western blot analyses indicated that RQCD1-specific shRNAs (shRNA\#1 and shRNA\#2) significantly suppressed RQCD1 expression while no change was observed in the MOCK-transfected cells (Fig. 2A). We then performed cell-proliferation and colony formation assays, and found that introduction of shRNA\#1 
(A)

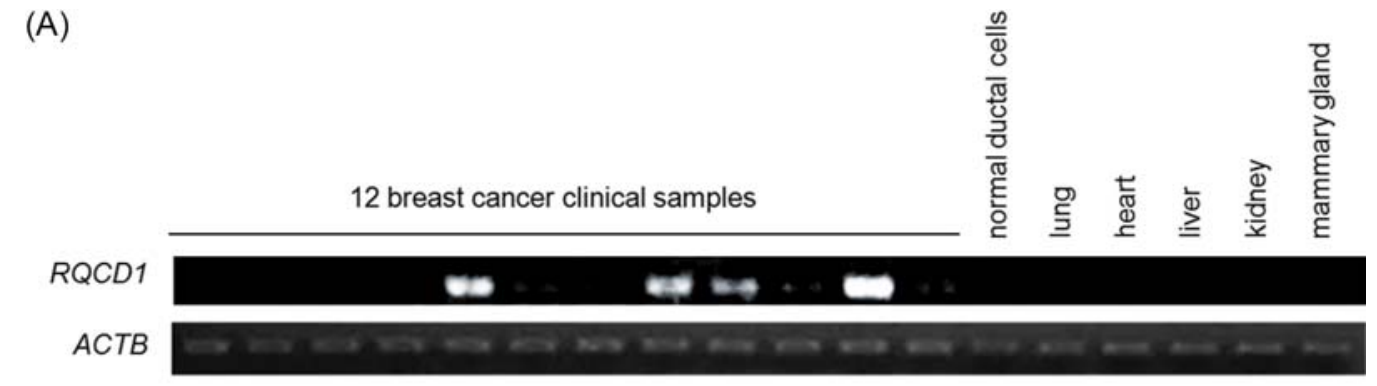

(B)

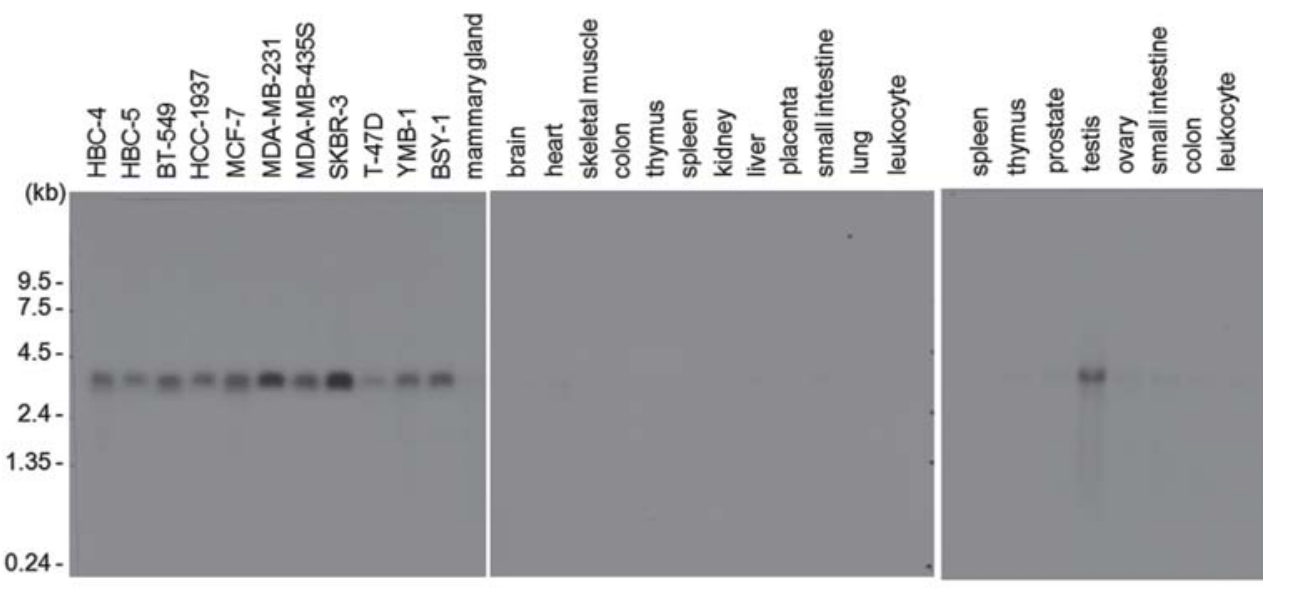

(C)
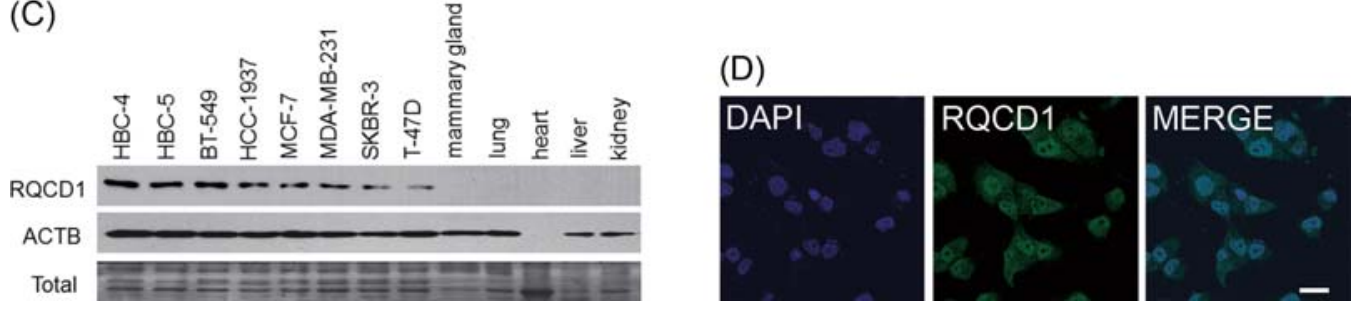

Figure 1. Expression levels of $R Q C D 1$ in clinical breast cancer samples, breast cancer cell lines and human normal tissues. (A) Semi-quantitative RT-PCR for 12 breast cancer clinical samples and human normal tissues including normal mammary ductal cells, whole mammary gland, lung, heart, liver and kidney. $\beta$-actin (ACTB) was used as an internal control. (B) Northern blot analysis for 11 breast cancer cell lines and human normal multiple tissues with a [ $\left.\alpha^{32} \mathrm{P}\right]-$ dCTP-labeled $R Q C D 1 \mathrm{cDNA}$ fragment as a probe. For breast cancer cell lines and human normal mammary gland, $1 \mu \mathrm{g}$ each of mRNA was applied to each lane. For human multiple normal tissues, $2 \mu \mathrm{g}$ each of mRNA was applied to each lane. (C) Western blotting of RQCD1 for breast cancer cell lines and human normal tissues with purified anti-RQCD1 polyclonal antibody. Five-microgram each of total protein was applied to each lane. Equal amount of loading proteins were confirmed by staining nitrocellulose membrane with Ponceou S. (D) Immunocytostaining of RQCD1 in BT-549 cells. RQCD1 was probed with anti-RQCD1 polyclonal antibody and Alexa-488 (green), and cell nuclear was counterstained with DAPI (blue). Scale bar indicates $20 \mu \mathrm{m}$.

and shRNA\#2 constructs significantly suppressed growth of both BT-549 and HBC-4 cells (BT-549: shRNA\#1, P=0.004

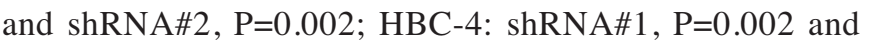
shRNA\#2, $\mathrm{P}=0.002$; Student's t-test), in concordance with the results of knockdown effect of the transcript (Fig. 2A). To further confirm the growth-promoting effect of RQCD1, we established three independent HEK293 derivative cells that stably expressed exogenous RQCD1 at high level (stable-1, -2 and -3) compared to parental HEK293 (Fig. 2B). Subsequent cell proliferation assay revealed that the three RQCD1-stable cells (stable-1, -2 and -3) grew significantly much faster than those transfected with mock plasmid (mock-1, -2 and -3; Fig. 2B right panel), indicating an oncogenic role of RQCD1 overexpression.

Identification of molecules interacting with RQCD1. To further investigate its biological function, we searched for a protein(s) interacting with RQCD1 protein in breast cancer cells by GST-pull down assay using the N-terminally GSTfused full-length RQCD1 recombinant protein (GST-RQCD1) and mass spectrometric analysis (see Materials and methods). Comparison of silver staining patterns of SDS-PAGE gels containing the pulled-down proteins identified two proteins, approximately at 140 and $160 \mathrm{kDa}$ specifically in the lane corresponding to proteins pulled-down with GST-RQCD1 protein (data not shown). Mass spectrometric analysis indicated these 140 and $160 \mathrm{kDa}$ proteins to be Grb10interacting GYF protein 1 (GIGYF1) and 2 (GIGYF2), respectively, which were previously indicated their involvement in the PI3K/Akt signaling pathway (16). Subsequently, to confirm the interaction between RQCD1 and GIGYF1/ GIGYF2, we performed co-immunoprecipitation assay (see Materials and methods). Flag-tagged RQCD1 (Flag-RQCD1), and HA-tagged GIGYF1 or GIGYF2 (HA-GIGYF1, HAGIGYF2) constructs were co-transfected into HEK-293T cells, and the cell lysates were immunoprecipitated with anti- 
(A)
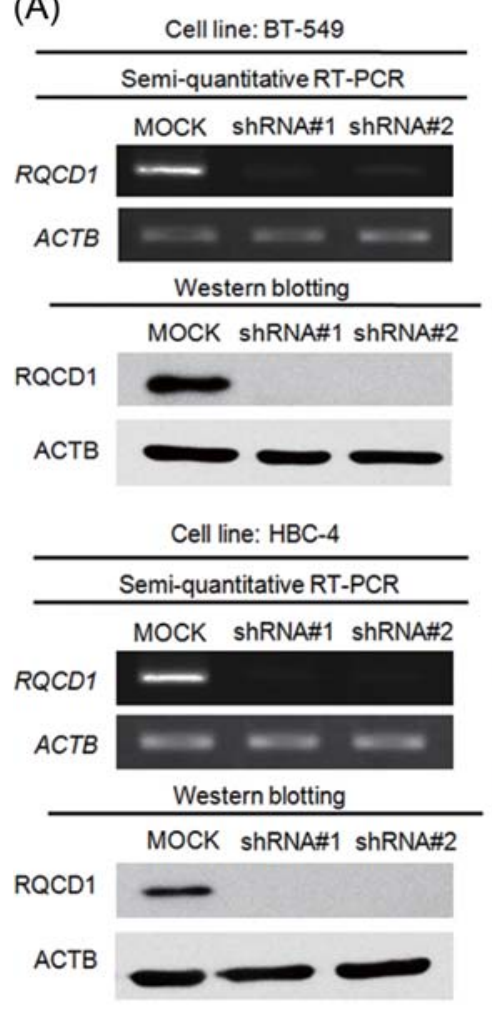

(B)

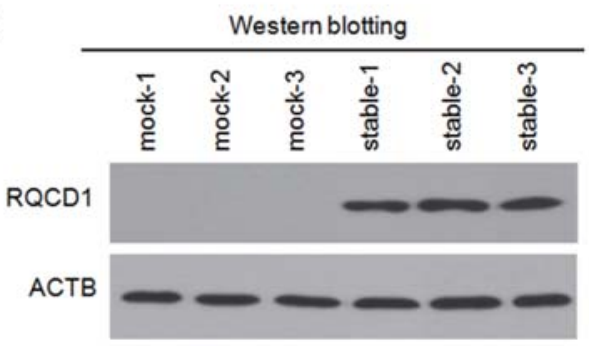

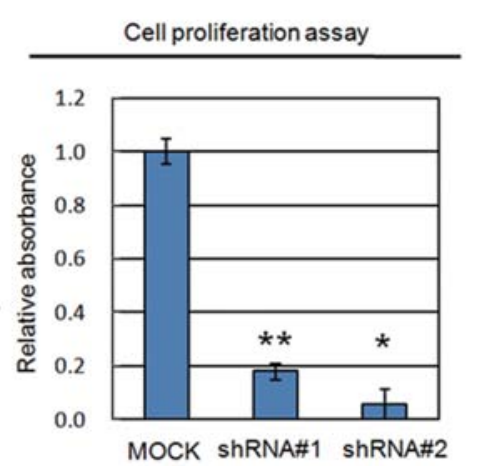
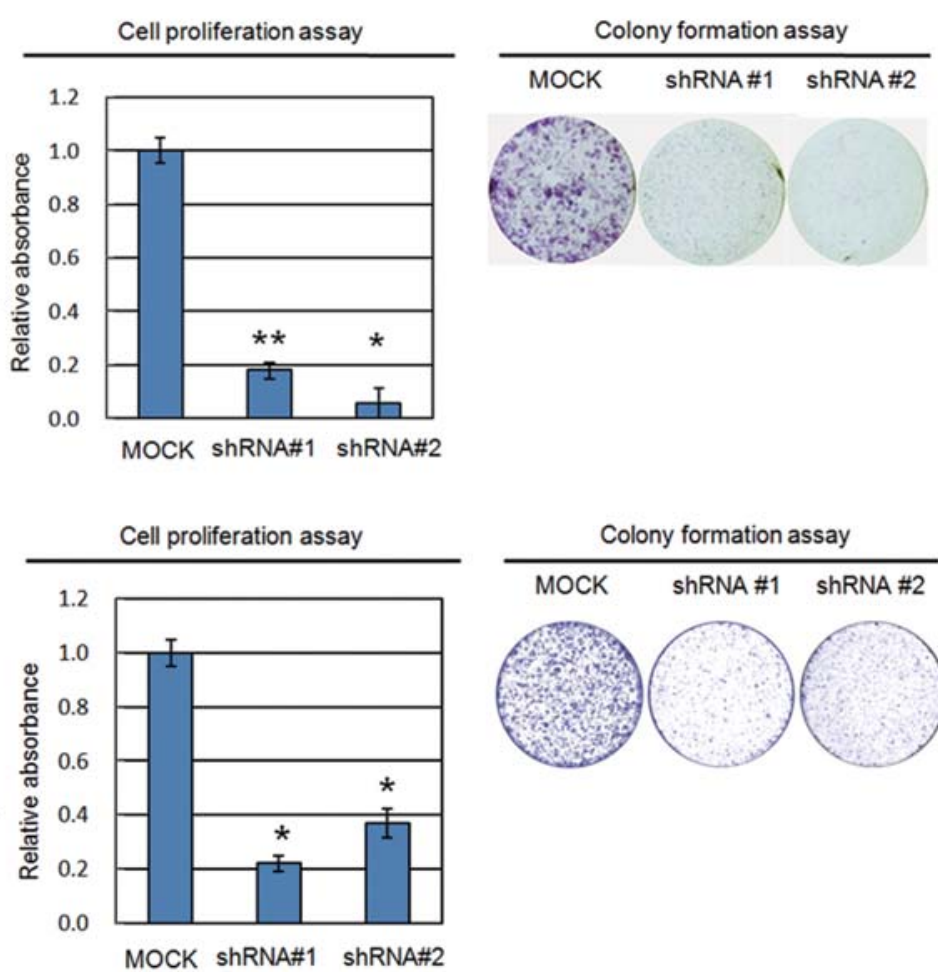

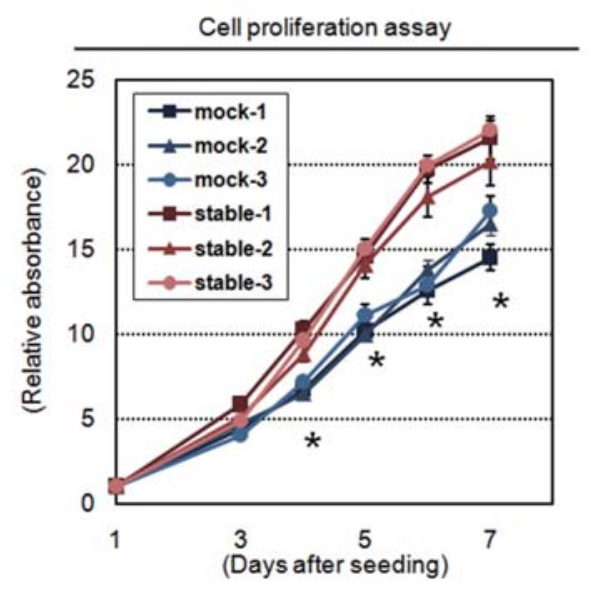

Figure 2. Effect of RQCD1 on cell growth. (A) Effect of RNAi on growth of breast caner cell lines. shRNA expression vectors specific to RQCD1 transcript (\#1 or \#2) and a mock shRNA expression vector (mock) were transfected into BT-549 and HBC-4 cells, respectively. Knockdown effect of RQCD1 was examined by semi-quantitative RT-PCR and Western blot analyses. Cell proliferation assay and colony formation assay were performed for evaluation of knockdown effect on cell growth. Columns, average of three independent experiments; bars, $\pm \mathrm{SE}$. ${ }^{*} \mathrm{P}=0.002$ and ${ }^{* *} \mathrm{P}=0.004$ by Student's t-test compared to mock transfected cells. (B) Growth rate of HEK293 cells in which RQCD1 was stably expressing. Western blotting was performed for three independent clones of HEK293 derivative cells expressing RQCD1 (stable-1, -2 and -3) and those transfected with mock vector (mock-1, -2 and -3). Each cell line was seeded at $0.4 \times 10^{5}$ cells to 6 -well plate, and 7 days after seeding, cell proliferation assay was performed. X-axis, day points after seeding; Y-axis, fold increase in cell number from the first day. Points, an average of three independent experiments; bars, \pm SE; * $P<0.0001$ by Student's t-test.

Flag antibody. Immunoblotting of the precipitates with antiHA antibodies suggested co-immunoprecipitation of FlagRQCD1 with HA-GIGYF1 or HA-GIGYF2. Conversely, we also carried out immunoprecipitation with anti-HA antibody and subsequent immunoblotting of precipitates with antiFlag antibody, and confirmed their co-immunoprecipitation (Fig. 3A). Then, we examined the transcriptional levels of GIGYF1 and GIGYF2 in breast cancer cell lines by semiquantitative RT-PCR, and found that GIGYF1 and GIGYF2 were also up-regulated in all breast cancer cell lines examined, compared with normal mammary gland (Fig. 3B). We further examined the subcellular localization of these proteins in breast cancer cells, BT-549, by immunocytochemical staining, and detected HA-GIGYF1 and HA-GIGYF2 proteins in cytoplasm, and partially colocalized with endogenous RQCD1.

Involvement of RQCD1 in Akt-signaling pathway. Since overexpression of GIGYF1 and GIGYF2 was reported to activate PI3K/Akt signaling pathway in mouse embryonic fibroblasts that were transfected with the IGF-I receptor (16), we examined whether RQCD1, GIGYF1 and GIGYF2 could effect on the Akt activity. The phosphorylation of Akt at 
(A)

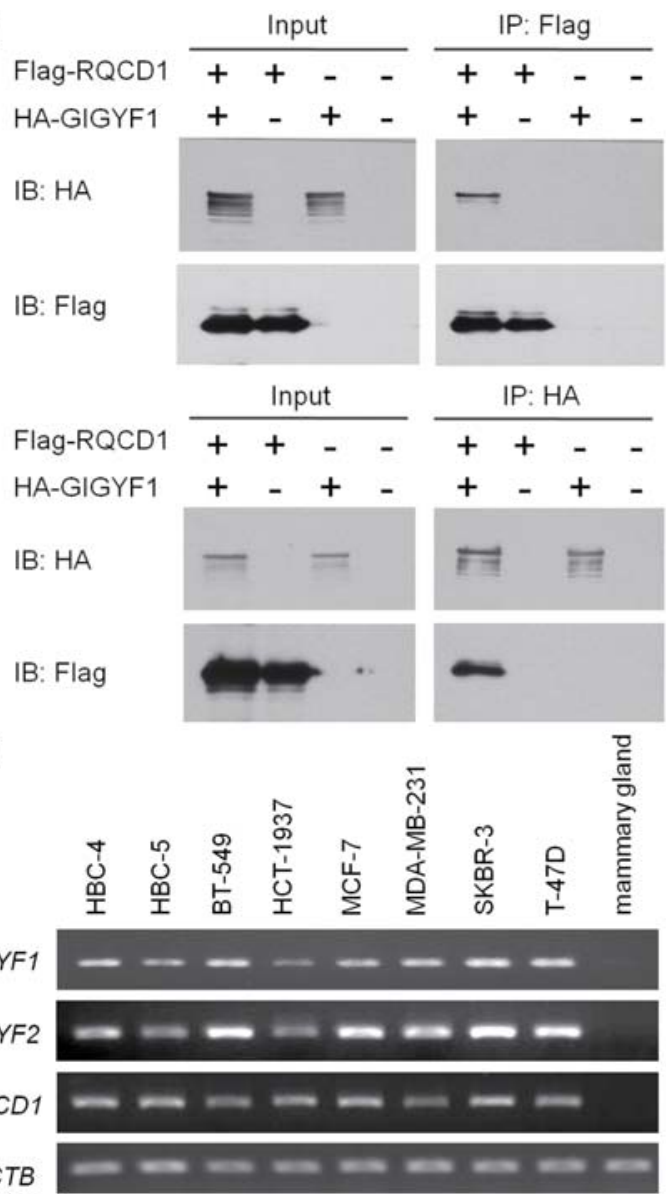

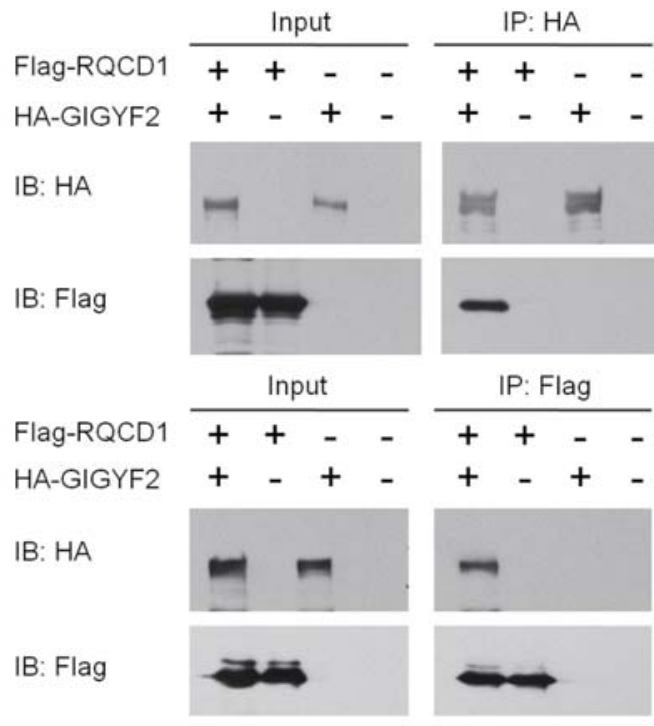

(C)

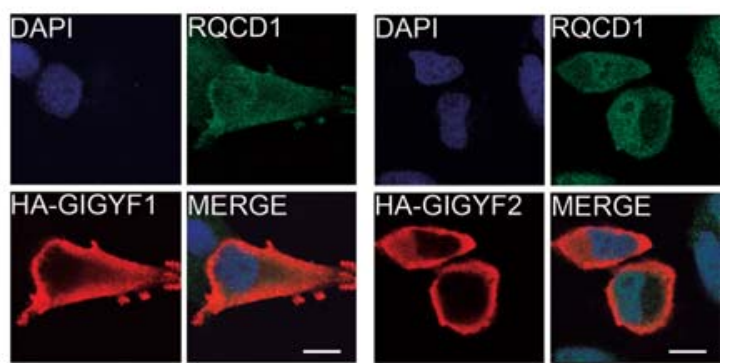

Figure 3. Interaction and co-localization of RQCD1 with GIGYF1 or GIGYF2. (A) Co-immunoprecipitation assay for Flag-RQCD1 and HA-GIGYF1 or HAGIGYF2 in HEK293T cells. Immunoprecipitation by anti-Flag or anti-HA agarose was performed at $36 \mathrm{~h}$ after the co-transfection of Flag-RQCD1, and HAGIGYF1 or HA-GIGYF2. Precipitated proteins were competitively eluted with 3XFlag peptide or HA peptide. Subsequently, Western blotting was performed for detection of input controls and peptide-eluted samples. (B) Expression levels of GIGYF1, GIGYF2 and RQCD1 in breast cancer cell lines and normal mammary gland were examined by semi-quantitative RT-PCR. ACTB served as an internal control. (C) Immunocytostaining of exogenously-expressed HAGIGYF1 and HA-GIGYF2 in BT-549 cells. Thirty-six hours after transfection to BT-549, the cells were fixed, and HA-GIGYF1 (red), HA-GIGYF2 (red), and endogenous RQCD1 (green) were immunostained. Nuclei were counterstained with DAPI (blue). Scale bar indicates $10 \mu \mathrm{m}$.

Ser 473 in its carboxyl-terminal hydrophobic motif is known to be a representative marker for activation of Akt (20-22). Therefore, we first performed Western blotting with anti-Akt and anti-phospho-Akt (Ser 473) antibodies to examine the Akt activity status in breast cancer cells, BT-549, HBC-5 and HCC-1937, which showed a high level of RQCD1 expression (Fig. 1C). The results showed that the high level of phosphorylation of Akt at Ser 473 was clearly observed in all breast cancer cells even in the absence of the serum stimulation, while its phosphorylation was abolished in normal ductal epithelial cell-derived MCF-10A in the serum-depletion condition (Fig. 4A), indicating that Akt is constitutively activated in these breast cancer cells. We then investigated the knockdown effects of RQCD1, GIGYF1 or GIGYF2 expression by siRNA treatments on the Akt phosphorylation level, and found that treatment of each siRNA against either RQCD1, GIGYF1 or GIGYF2 into BT-549 cells caused the significant reduction of phosphorylation level of Akt without alteration of total Akt protein level (Fig 4B and D). We also observed a similar effect on the Akt activity by the RQCD1siRNA treatment in the other breast cancer cell lines, HBC-5 and HCC-1937 (Fig. 4C and D).

\section{Discussion}

Molecular targeting drugs for breast cancer therapy have contributed to reduction in motility rate and improvement in QOL of patients in the last two decades $(1,2)$. However, the proportion of patients showing good response to presently available treatments is still limited particularly for the patients at advanced stages or those with triple-negative breast cancer (23). Toward identification of molecular targets for drug development, we had analyzed the detailed gene expression profiles of 81 clinical breast cancer cells (6) and 29 normal human tissues (24) for selecting genes that were up-regulated specifically in breast cancer cells in combination with experiments screening for knock down effects by means of the RNA interference system. On the basis of this approach, we found RQCD1 to be up-regulated frequently in clinical breast cancer samples as well as breast cancer cell lines, while its expression was very low in normal human tissues except the testis. These results indicated RQCD1 as a novel cancertestis antigen. Furthermore, we demonstrated that knockdown of RQCD1 expression resulted in significant growth suppression of breast cancer cells and that introduction of 

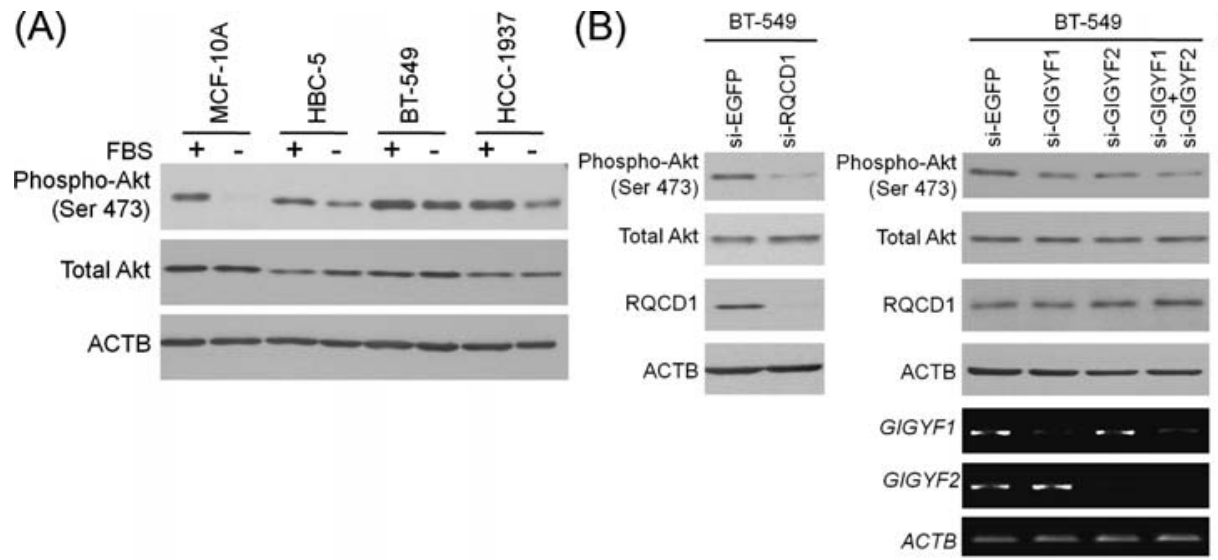

(C)
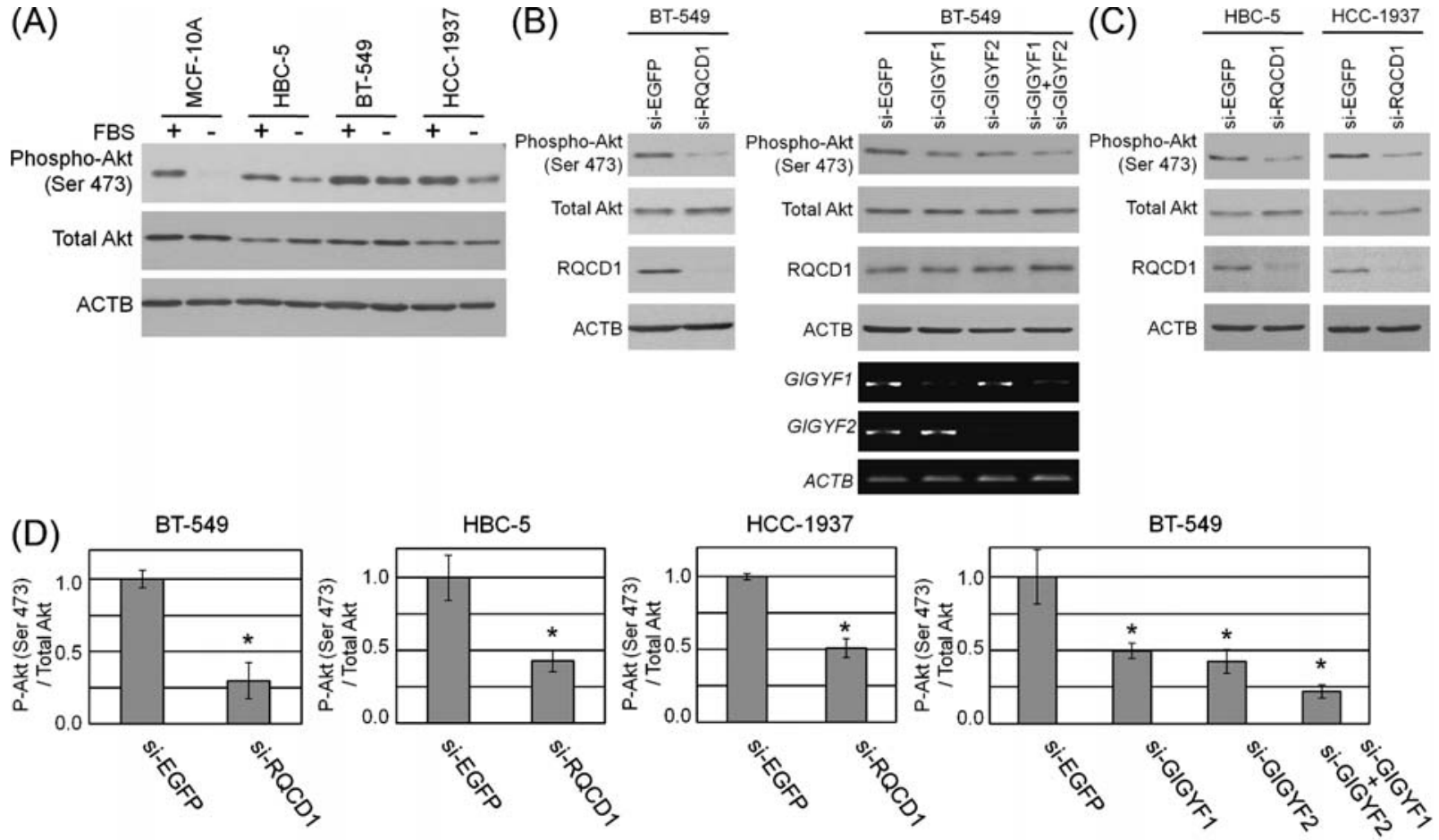

Figure 4. Effect on Akt activity by knockdown of RQCD1, GIGYF1 or GIGYF2 in breast cancer cells. (A) Effect on Akt activity in breast cancer cells under presence or absence of serum treatment. MCF-10A, BT-549, HBC-5 and HCC-1937 cells were cultured in each appropriate culture medium with or without FBS and growth factors for $24 \mathrm{~h}$, and then Akt activity was analyzed by Western blotting with anti-Akt and anti-phospho-Akt (Ser 473) antibodies. (B) The total amount of Akt and its phosphorylation level were examined by Western blotting with anti-Akt and anti-phospho-Akt (Ser 473 ) antibodies at 72 h after transfection of siRNA against RQCD1 (left panels), GIGYF1 or GIGYF2 (right panels) in BT-549 cells. Cells were cultured in serum-depleted medium for $24 \mathrm{~h}$ before harvesting. Knockdown effects of GIGYF1 and GIGYF2 were confirmed by semi-quantitative RT-PCR. (C) The total amount of Akt and its phosphorylation level were examined by Western blotting with anti-Akt and anti-phospho-Akt (Ser 473) antibodies at $72 \mathrm{~h}$ after knockdown of RQCD1 in HBC-5 (left panels) and HCC-1937 cells (right panels). Cells were cultured in serum-depleted medium for $24 \mathrm{~h}$ before harvesting. (D) Akt activity in each breast cancer cell line was quantitatively evaluated by ratio of phospho-Akt (Ser 473)/total Akt signal intensity by densitometric analysis of ECL signals using Image J (19). Assays were carried out three times. Columns, average of three independent analysis; bars, $\pm \mathrm{SE}$; ${ }^{*} \mathrm{P}<0.05$ by Student's t-test, compared to si-EGFP treated cells.

RQCD1 into HEK293 cells significantly promoted the cell growth, implying that RQCD1 could serve as a valuable target for development of anticancer agents or cancer peptide vaccine for breast cancer.

RQCD1, a protein evolutionarily conserved among eukaryotes, was first identified as a crucial factor for regulation of differentiation in nitrogen-starved fission yeast; yeast cells lacking of RQCD1 were reported to be sterile when they were cultured in the nitrogen-starvation condition (14). Furthermore, the murine homolog of RQCD1 was reported as a transcriptional cofactor that mediated retinoic acid-induced differentiation and also to be an erythropoietin-responsive gene potentially involved in development of hematopoietic cell $(15,25)$. However, since its biological roles in tumorigenesis have not been investigated, we searched for interacting proteins of RQCD1 and identified the interaction of RQCD1 with both GIGYF1 and GIGYF2 proteins that were reported to be involved in activation of the PI3K/Akt signaling pathway (16). We further confirmed that knockdown of RQCD 1, GIGYF1 or GIGYF2 by siRNA treatment resulted in reduction of the phosphorylation level of Akt at Ser 473, that is known to be a marker of its activation (20-22), in breast cancer cells in which these genes was overexpressed, indicating that RQCD1 might function in the constitutive activation of
Akt in breast cancer cells through the interaction with GIGYF1 and GIGYF2.

Our findings suggest that RQCD1 is overexpressed specifically in breast cancer cells, and plays an important role in cell growth of breast cancer. In addition, this gene product might be involved in the regulation of constitutive activation of Akt through physiological interaction with GIGYF1 and GIGYF2 in several cellular contexts. Consequently, our findings in this study not only contribute to understanding of mammary carcinogenesis, but also provide RQCD1 as a potential candidate for development of molecular target drug against breast cancer.

\section{Acknowledgements}

We greatly thank Ms. Kyoko Kijima, Yoshiko Fujisawa and Kie Naito for technical support.

\section{References}

1. Parkin DM, Bray F and Ferlay J: Global cancer statistics, 2002. CA Cancer J Clin 55: 74-108, 2005.

2. Veronesi U, Boyle P, Goldhirsch A, Orecchia R and Viale G: Breast cancer. Lancet 365: 1727-1741, 2005.

3. Bange J, Zwick E and Ullrich A: Molecular targets for breast cancer therapy and prevention. Nat Med 7: 548-552, 2001. 
4. Cooper CS: Applications of microarray technology in breast cancer research. Breast Cancer Res 3: 158-175, 2001.

5. Slonim DK: From patterns to pathways: gene expression data analysis comes of age. Nat Genet 32: 502-508, 2002.

6. Nishidate T, Katagiri T, Lin ML, et al: Genome-wide geneexpression profiles of breast-cancer cells purified with laser microbeam microdissection: identification of genes associated with progression and metastasis. Int J Oncol 25: 797-819, 2004.

7. Park JH, Lin ML, Nishidate T, Nakamura Y and Katagiri T: PDZ-binding kinase/T-LAK cell-originated protein kinase, a putative cancer/testis antigen with an oncogenic activity in breast cancer. Cancer Res 66: 9186-9195, 2006.

8. Shimo A, Nishidate T, Ohta T, Fukuda M, Nakamura Y and Katagiri T: Elevated expression of protein regulator of cytokinesis 1 , involved in the growth of breast cancer cells. Cancer Sci 98: 174-181, 2007.

9. Shimo A, Tanikawa C, Nishidate T, et al: Involvement of kinesin family member $2 \mathrm{C} /$ mitotic centromere-associated kinesin overexpression in mammary carcinogenesis. Cancer Sci 99: 62-70, 2008.

10. Lin ML, Park JH, Nishidate T, Nakamura Y and Katagiri T: Involvement of maternal embryonic leucine zipper kinase (MELK) in mammary carcinogenesis through interaction with $\mathrm{Bcl}-\mathrm{G}$, a pro-apoptotic member of the Bcl-2 family. Breast Cancer Res 9: R17, 2007.

11. Ueki T, Nishidate T, Park JH, et al: Involvement of elevated expression of multiple cell-cycle regulator, DTL/RAMP (denticleless/RA-regulated nuclear matrix associated protein), in the growth of breast cancer cells. Oncogene 27: 5672-5683, 2008.

12. Lin ML, Fukukawa C, Park JH, et al: Involvement of overexpression of G-patch domain containing 2 in breast carcinogenesis. Cancer Sci (In press).

13. Kim JW, Akiyama M, Park JH, et al: Activation of an estrogen/estrogen receptor signaling by BIG3 through its inhibitory effect on nuclear transport of PHB2/REA in breast cancer. Cancer Sci (In press).

14. Okazaki N, Okazaki K, Watanabe Y, Kato-Hayashi M, Yamamoto $\mathrm{M}$ and Okayama $\mathrm{H}$ : Novel factor highly conserved among eukaryotes controls sexual development in fission yeast. Mol Cell Biol 18: 887-895, 1998.
15. Hiroi N, Ito T, Yamamoto H, Ochiya T, Jinno S and Okayama H: Mammalian Red1 is a novel transcriptional cofactor that mediated retinoic acid-induced cell differentiation. EMBO J 21: 5235-5244, 2002

16. Giovannone B, Lee E, Laviola L, Giorgino F, Cleveland KA and Smith RJ: Two novel proteins that are linked to insulin-like growth factor (IGF-1) receptors by the Grb10 adapter and modulate IGF-1 signaling. J Biol Chem 34: 31564-31573, 2003.

17. Katagiri T, Ozaki K, Fujiwara T, et al: Cloning, expression and chromosome mapping of adducin-like 70 (ADDL), a human cDNA highly homologous to human erythrocyte adducin. Cytogenet Cell Genet 4: 90-95, 1996.

18. Shimokawa T, Furukawa Y, Sakai M, Li M, Miwa N, Lin YM and Nakamura Y: Involvement of the FGF18 gene in colorectal carcinogenesis, as a novel downstream target of the B-catenin/ T-cell factor complex. Cancer Res 63: 6116-6120, 2003.

19. Abramoff MD, Magelhaes PJ and Ram SJ: Image processing with image J. Biophotonics Int 11: 36-42, 2004.

20. Alessi DR, Andjelkovic M, Caudwell B, Cron P, Morrice N, Cohen $\mathrm{P}$ and Hemmings BA: Mechanism of activation of protein kinase B by insulin and IGF-1. EMBO J 15: 6541-6551, 1996.

21. Yang J, Cron P, Thompson V, Good VM, Hess D, Hemmings BA and Barford D: Molecular mechanism for the regulation of protein kinase B/Akt by hydrophobic motif phosphorylation. Mol Cell 9: 1227-1240, 2002.

22. Scheid MP, Marignani PA and Woodgett JR: Multiple phosphoinositide 3-kinase-dependent steps in activation of protein kinase B. Mol Cell Biol 22: 6247-6260, 2002.

23. Bange J, Zwick E and Ullrich A: Molecular targets for breast cancer therapy and prevention. Nat Med 7: 548-552, 2001.

24. Saito-Hisaminato A, Katagiri T, Kakiuchi S, Nakamura T, Tsunoda $\mathrm{T}$ and Nakamura Y: Genome-wide profiling of gene expression in 29 normal human tissues with a cDNA microarray. DNA Res 9: 35-45, 2002.

25. Gregory RC, Lord KA, Panek LB, Gaines P, Dillon SB and Wojchowski DM: Subtraction cloning and initial characterization of novel Epo-immediate response genes. Cytokine 12: 845-857, 2000 . 